\title{
How to increase CRM user adoption - Interview with Inna Proshkina of InvisibleCRM
}

\section{Inna Proshkina}

marketing director of InvisibleCRM, is responsible for the development and performance of the company's overall marketing strategy and corporate communications. She also drives the company's new business development and takes care of partner relations and contributes in numerous ways to the positioning and the promotion of InvisibleCRM's products on the US and European markets. To ensure InvisibleCRM's solid positions, she also collaborates with leading analytical agencies. Prior to InvisibleCRM, Proshkina had worked as a marketing director for Vested Development, a software engineering company. She got her Master of Science degree with honors from the Russian State University of Humanities.

\section{Keywords: outlook integration, user adoption, CRM, document management, InvisibleCRM, Sales Force}

Abstract The biggest challenge with business applications is actually getting users to use it. Michael Moon spoke with Inna Proshkina of InvisibleCRM on their innovative technology that bridges the gap between corporate systems and productivity tools to drive user efficiency and adoption.

Journal of Digital Asset Management (2008) 4, 225-233. doi:10.1057/dam.2008.24

MM: We're here with InvisibleCRM. Inna, please give us a brief introduction of yourself. IP: My name is Inna Proshkina. I'm marketing director with InvisibleCRM. I have worked with InvisibleCRM from the very beginning, which was back in 2005 .

I'm responsible for marketing strategy development and corporate communications. Also, I collaborate with partners, customers, media and analytical agencies.

Prior to InvisibleCRM, I worked as a marketing director for Vested Development a software outsourcing company.

MM: Excellent.

Can you give us a bit of a background in terms of InvisibleCRM, how large it is and some of the kinds of clients that it serves? IP: InvisibleCRM is not large at all - we have about 30 employees. InvisibleCRM is headquartered in San Mateo, California with a sales and support office there. Our development centers are located in Moscow, Russia - and also in Kiev, Ukraine.

Regarding our background and how the idea of InvisibleCRM was born, let me give you an example that illustrates that.

If we look at CRM applications, at its core is Sales Force automation - leads, opportunities, contacts, pipeline reports and so on. Sales Force automation users are sales executives. They're strong personalities that do not want to concern themselves with all those CRM realities.

That was quite unfortunate for our sales and corporate management who needed to know what our pipeline looked like, and how to manage investor expectations.

MM: So you know, Inna - one of the conversations that has emerged out of Sales Force automation and CRM has been the classic conflict between the tactical needs of sales personnel in the field, or sales personnel within an inside sales team.

Then, sales management - which is trying to not only understand what's in the sales pipeline in terms of revenue forecasts - but also to understand if the salesmen are making the number and the kinds of calls they need to make.

Therein lies the conflict. The sales people really only want to do as much data entry and working with data as is necessary to sell something. Whereas sales management is looking for larger management of an overall business process. Did I summarize that right?

IP: Yes. Exactly.

The key challenge for all companies that aim to make use of Sales Force automation is user adoption. Because if a sales team is not using 
CRM constantly and consistently, then management will not see the value of this system. Right?

MM: Right.

IP: So, how do we make our users more effective at using these CRM systems that organizations throw at them?

We started thinking about how our sales reps think. We came up with the conclusion that they all have notebooks, and all those applications they use to work documents, spreadsheets, presentations, tasks and calendar are personal Information Managers. In 95 per cent of the cases it is MS Outlook or Lotus Notes and MS Office. There is a corporate reality that says that there are 200 million MS Outlook users in the world! That brought us to a very simple conclusion, which is at the foundation of InvisibleCRM philosophy: "Don't change people, but change the software."

If we want our sales reps to use CRM effectively, we need to serve it to them inside of Outlook. So they don't even see the difference between the two applications: CRM and Outlook. And also to leverage that back-end CRM and our integration to generate value. Which is exactly what InvisibleCRM is - a suite of products to seamlessly meld back-end applications into the way users work with Outlook and Windows.

We started with Salesforce.com, since we liked it. We saw a big market opportunity, and we happened to be a Sales Force customer ourselves. But actually, almost everything in our product line and our design philosophy applies to many other kinds of enterprise applications — such as document management applications.

MM: Inna, would you take us through the various kinds of field executives who have a need for CRM and/or Sales Force automation? Frontline sales executives, typically in a B2B or B2C type of business.

IP: Well, there are two types of salespeople. The first group would include sales executives and sales managers. They mostly use the reporting capabilities of CRM. Of course, they need to get actual and accurate data from their sales reps to know what's going on with their pipeline and take immediate action if needed.
The second group is sales reps who work in the field - or corporate sales reps. They need to enter different types of information into the CRM system, to provide the data for decisionmaking to their sales managers. Half of the salespeople usually work in the field. They don't always have a connection to the CRM system or time to enter data.

For example after hot trade shows and events, a sales rep needs to enter contact information (leads, meeting minutes and follow up tasks) into the CRM system. Normally he cannot liberate the time that he spends, for example, on the plane, because he is not connected to CRM. InvisibleCRM provides a fast gateway to the CRM system through MS Outlook. A sales rep enters a new contact in MS Outlook and once connected, this information is synced with CRM. The same applies to all the changes made to other CRM records and calendar. MM: It seems to me that because the contact information lives in Outlook, it is therefore a persistent fat client in a database. It also seems to me that there are other services like Plaxo a service that allows me to manage any changes of address.

IP: Yes. That's right.

MM: Could you speak to that application as you understand it as a practitioner?

IP: Yes.

You can synchronize Plaxo and other contact management tools with CRM through MS Outlook using InvisibleCRM. For example, your customer updates the contact information in Plaxo. You receive the contact updates in your Outlook and then this information is synchronized with CRM.

Normally every business person works within an information system that consists of a corporate application, personal laptop and mobile phone. We got used to making appointments online, adding events in MS Outlook and then going to a meeting and checking details on the fly using the phone (address, cell number or contact's name). We want to live in the single information environment and not to worry about synchronization processes (Figure 1).

A user just needs to create a contact or save a document in one place and it is automatically distributed through CRM, MS Outlook or Office, and mobile phone. By the way, if you buy 
a new laptop or mobile phone, after the first synchronization you will have all your data at hand. MM: Let's go into more of the specifics of how InvisibleCRM works.

IP: Actually, InvisibleCRM implies three products. The first is CRM Outlook integration, which is called SalesDesktop. The second product is SalesFolder, a document sharing and delivery tool that synchronizes files between a corporate repository and a local subfolder on your desktop. The third product is

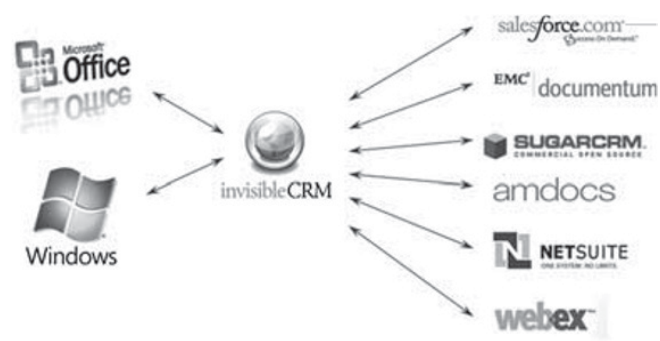

Figure 1: InvisibleCRM is a synchronization tool for business users. InvisibleCRM bridges the gap between business applications and productivity tools called SalesAlerts. That brings real-time notifications on important changes made in a corporate system.

Speaking about the first product SalesDesktop. SalesDesktop seamlessly unites the two essential applications for sales people: CRM and the business cornerstone, Microsoft Outlook, creating a fully featured sales workspace inside Outlook that works both in on and offline modes (Figure 2).

Users can enter information once, and SalesDesktop automatically and invisibly synchronizes the data with CRM. One click captures and saves e-mail to CRM easily and automatically. Users can take advantage of Outlook to synchronize customer information with handheld or PDA, and work with vital customer information anywhere and anytime, even offline.

MM: So the SalesDesktop works as a kind of plug-in to Microsoft Outlook, and also allows the Microsoft Outlook user to use Outlook as an interface to Salesforce.com. Did I understand that right?

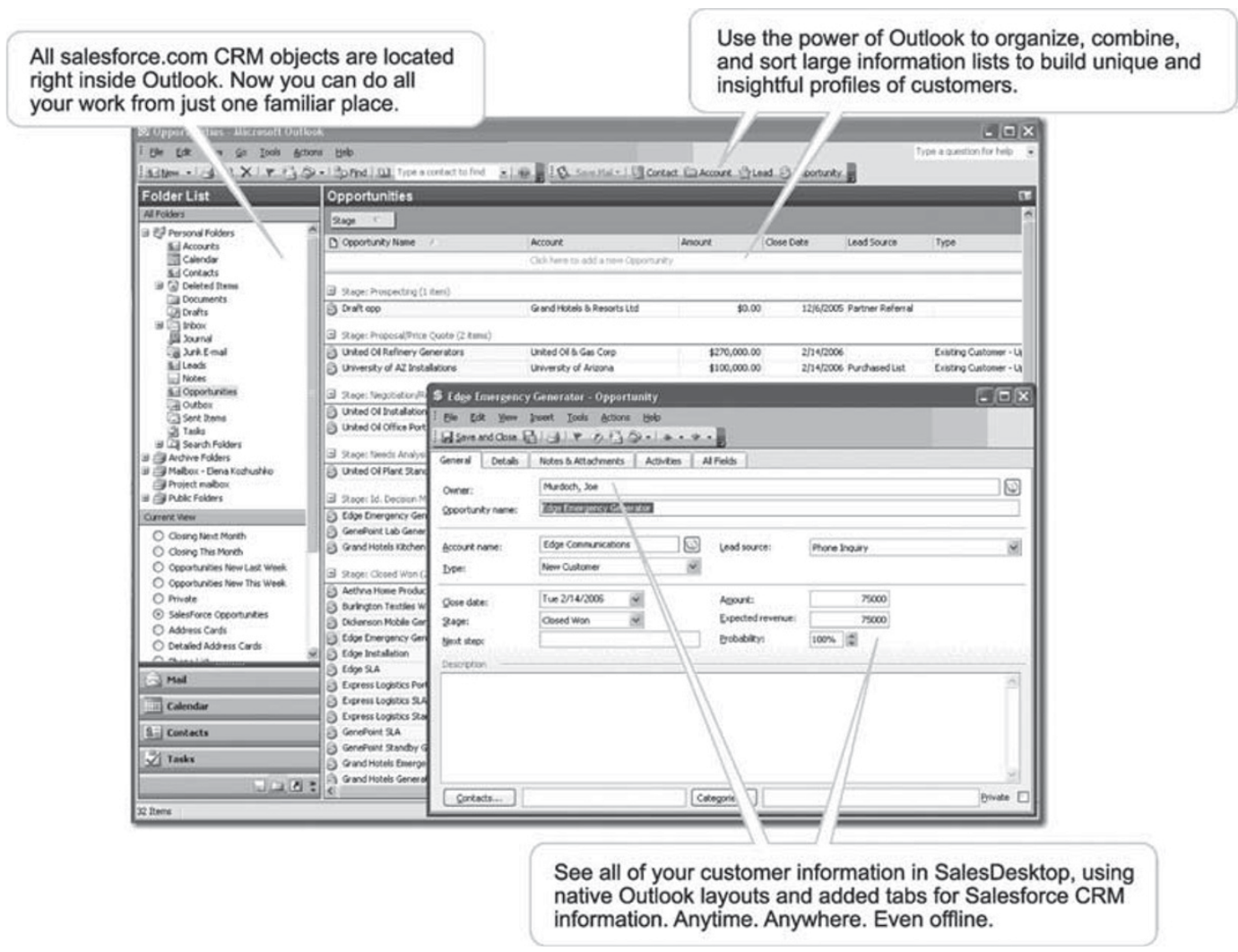

Figure 2: InvisibleCRM's SalesDesktop. SalesDesktop - the best CRM Outlook integration on the market 
IP: Yes. That's right.

MM: So then Salesforce.com becomes a shared database for a sales operation. Is that right?

IP: Well, I would say that SalesDesktop is an interface, and the data entry tool for sales reps.

But we left all business flows - such as converting leads to accounts and opportunities - or reporting functions on the CRM side. So the SalesDesktop is mostly a productivity tool for sales reps, rather than sales managers.

It saves time dramatically. For example, to keep a fuller history of customer interactions, a sales rep needs to attach every e-mail and activity with a customer to the CRM system. It requires extra efforts and time, and most sales reps just don't do it regularly. SalesDesktop automates this long and tedious to master process. They just need to select which contacts they want to track conversations with, and all the e-mails - incoming and outgoing — will be automatically attached as activities to the appropriate accounts and contacts within CRM.

Another example is that SalesDesktop unifies contacts, calendar and tasks. It means that users do not need to double-enter the same contacts, appointments and tasks twice - first in MS Outlook where they normally work, and then in CRM to share this information with their managers and colleagues. Once a record is created in Outlook, it automatically appears in CRM.

When a sales manager needs to see what's happening to this or that customer, he opens up a customer profile to see all the e-mails and other activities that were attached automatically to that customer.

MM: One last question about SalesDesktop. Does it interact at all with Microsoft Exchange — the server side part of the overall e-mail ecosystem of Outlook?

IP:Yes, SalesDesktop works with Microsoft Exchange as well.

MM: Is there any support for the Macintosh version of the Microsoft Suite?

IP: At the moment, we do not support that, but we might be supporting it sometime in the future.

MM: Sure. What about Microsoft SharePoint? IP: For Microsoft SharePoint, we have another product, which is called SalesFolder. It replicates MS SharePoint document repository structure and maps all documents to a local folder inside of "My Documents" folder (Figure 3).

Business users tend to store their files on laptops and are reluctant to upload them to the corporate system if it takes more than 2-3 clicks.

SalesFolder is an automatic document synchronization and delivery system that enables you to work with all of your documents anytime, even while disconnected. Using the same structure for documents found on a corporate system, SalesFolder builds a document repository in My SalesFolder on your desktop with all of your docs and attachments. It also enables you to work with all documents any time and add new files with drag and drop while all changes and additions are automatically synchronized and delivered to the desktop of other SalesFolder users. Let it be the Microsoft SharePoint server or any other documentmanagement system such as EMC Documentum, for example.

MM: Therefore, allowing for a more transparent and easy flow and/or synchronization of documents in and out of the SharePoint server. Is that right?

IP: That's right. Not only with Microsoft Sharepoint server, but also with any other document-management system.

Another very useful feature is tight integration with Microsoft Office. For example, if you have just created a document in PowerPoint or in Word, you can save it directly to the document repository through SalesFolder. MM: It seems to me that one way you could manage all of these various documents that are in an online area would be to simply build a spreadsheet and then put into the spreadsheet the hyperlinks of the various documents that are stored online.

IP: Users are not always online. And for busy business users who travel a lot, it's a great benefit to be able to work with documents while offline.

MM: Fair enough. Okay. What about the third product?

IP: SalesAlerts is the best way for you to stay on top of the changes to your customer or corporate information. It uses unobtrusive popup windows (like the MSN messenger) to notify you when changes have been made, which object has been changed, and by whom. With 


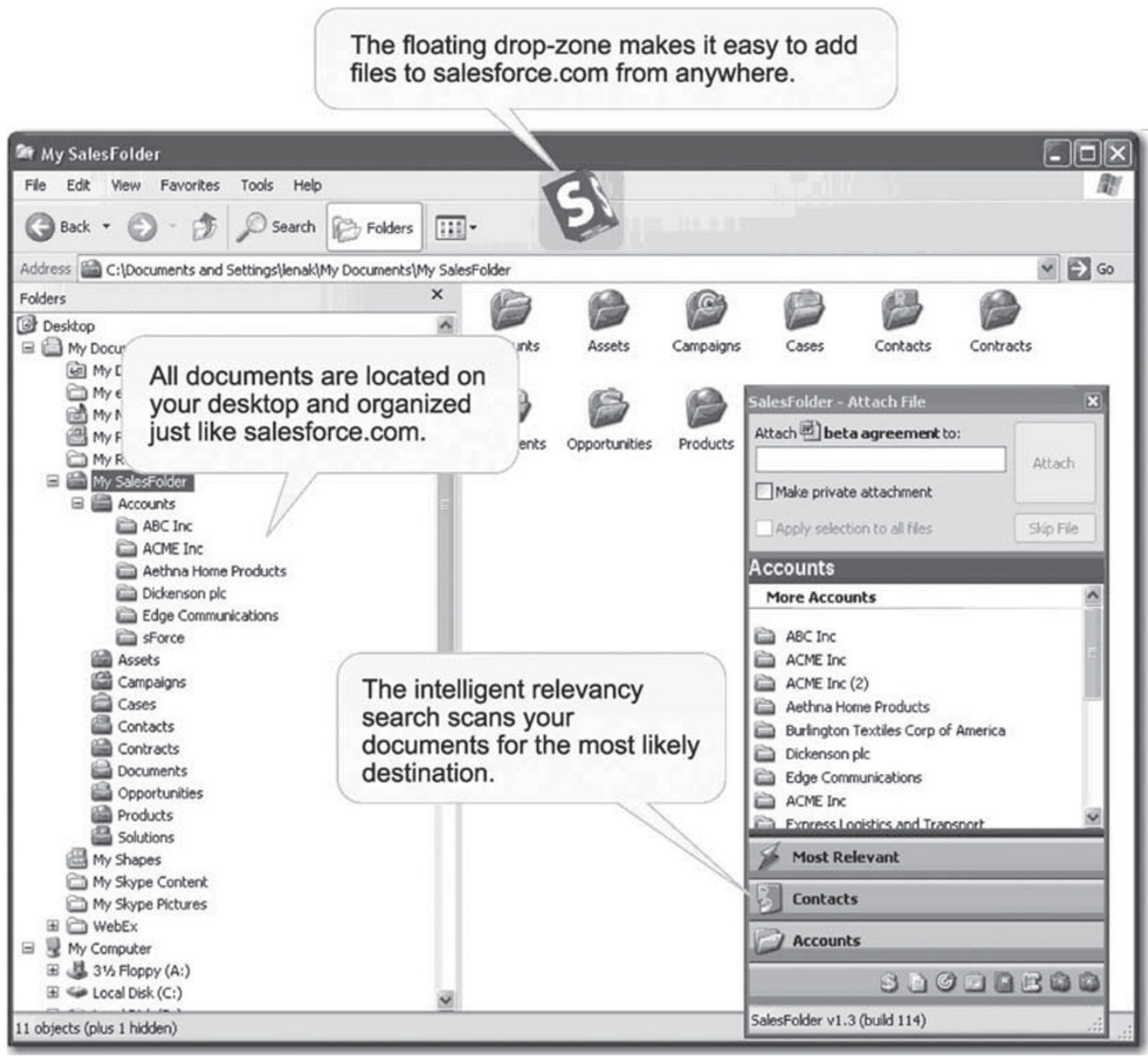

Figure 3: InvisibleCRM's SalesFolder. SalesFolder is an automatic document synchronization and delivery system

SalesAlerts, you can react to new opportunities or leads with just one click. You do not need to spend time browsing a CRM or other corporate system looking for updates (Figure 4).

MM: Simply a mouse-over on a particular notification would then reveal an underlying message associated with that notification. Is that right?

IP: Yes. That's right.

They can receive those updates in real time. Once a change is made, they receive a pop-up that shows what's been updated.

In our world it's crucial to react in time (for example, if a new lead is created in the system or a new support case is opened). SalesAlerts solves the main problem - they enable users to react immediately to those events that you are interested in and that your success depends on. The business value is faster business process and potentially more deals closed.

Alternatively, if managers do not want to be distracted from their important work with these pop-ups, they can open a SalesAlerts window that summarizes recent updates by person or by category.

MM: Let's shift gears now. Let's speak of the ways by which you measure value or ROI from these tools, and what kinds of results clients have reported. IP: Actually, last year, Nucleus Research did this kind of survey. They calculated that ROI for SalesDesktop is the first five hours of saved time for a sales person.

One of the goals of our application is to make a sales rep's life easier and to save their 


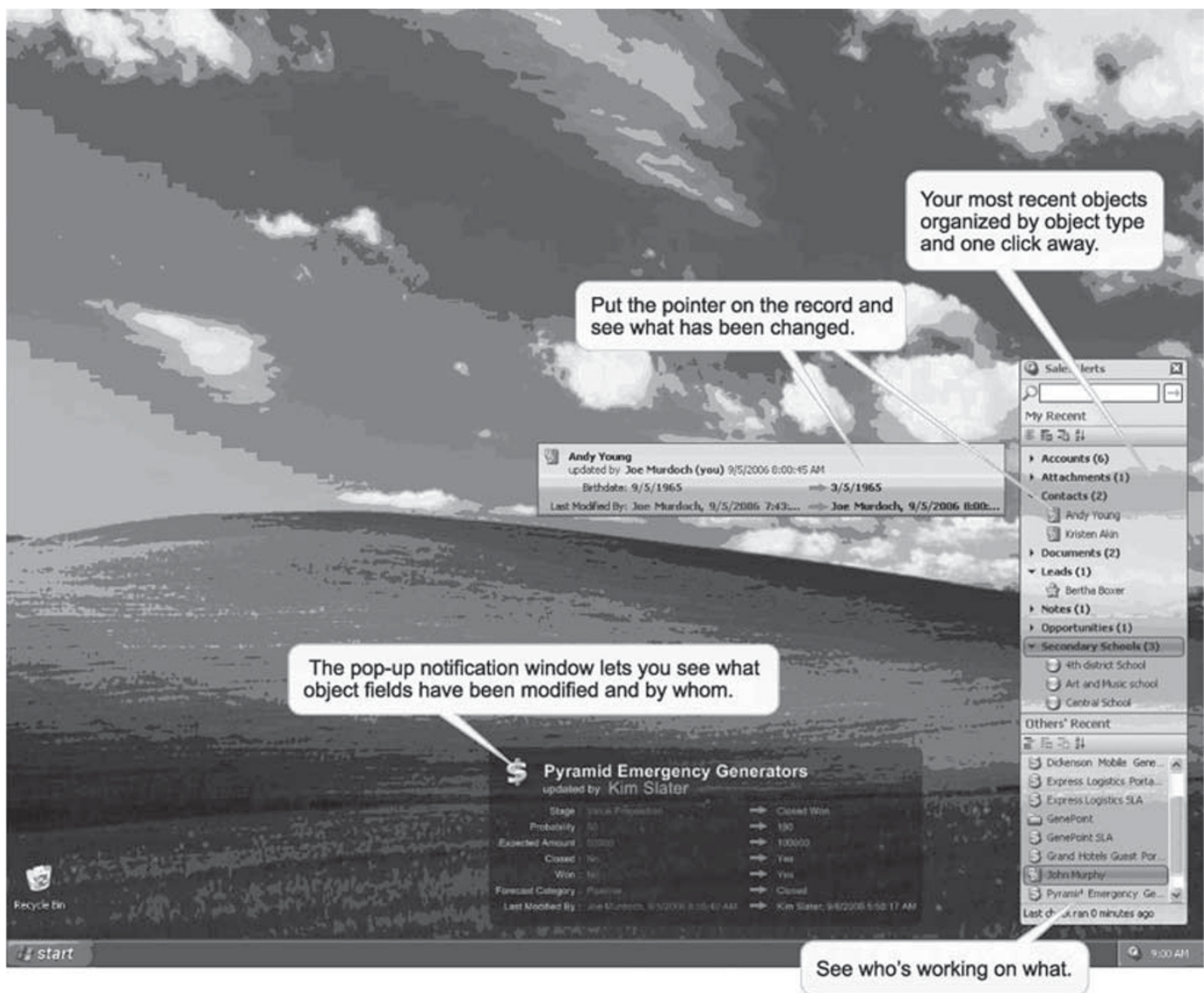

Figure 4: InvisibleCRM's SalesAlerts. SalesAlerts enables users to react immediately to important events in their corporate life

time. So when our application saves five hours of a sales rep's time, they pay back.

We have calculated that these five hours of saved time can be exceeded within the first two weeks of SalesDesktop usage.

MM: What other sorts of added value does using your system bring to a user or organization?

IP: Well, as I've mentioned previously, the key to successful CRM deployment is user adoption. Because if a sales team doesn't use CRM, then management doesn't take advantage of it. InvisibleCRM melds CRM into natural user environments that sales folks are used to working nowadays - MS Outlook and Office. It drives their productivity, since if they use Outlook they already use CRM. InvisibleCRM unifies contact, calendar and tasks, so a user needs to enter information once and it's automatically synced with CRM. Every e-mail is associated with the appropriate contact or account. So InvisibleCRM improves data quality and integrity. As a result, management gets what it wanted - clear visibility and forecasting (Figure 5).

\section{MM:Yes.}

Have you done any analysis as to the economic value of better contact data and/or the amount of money that an organization will typically spend to rebuild quality databases as opposed to simply having them from the beginning?

If I understand it right so far, first and foremost there's a certain economic value of mitigating nonadoption of CRM. There's a certain number of CRM deployments that will succeed because you've reduced the "hassle factor" of using it. That has a certain value.

The second area of value is that it saves a sales executive $\mathrm{X}$ number of hours per month as a function of this easier-to-use system. Generally, saving a sales executive five hours a month 


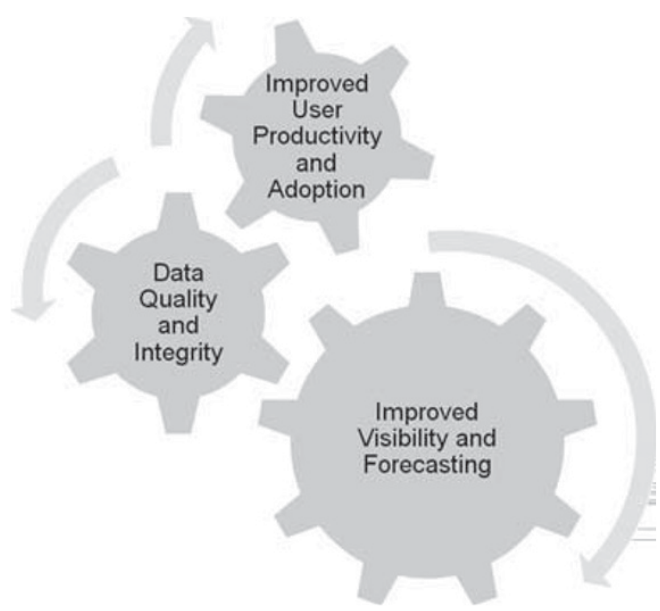

Figure 5: User Adoption is the key. InvisibleCRM provides organizations with a cost-effective way to drive user adoption and improve the value of their CRM initiative.

means - in practical terms, according to together research that I've seen — what translates into about two or three extra sales calls per week.

By the way - Harvard Business School did an extensive study on industrial sales processes and techniques and practices. They found that the fastest and most direct way of increasing sales — and more specifically, generating incremental sales - was to have a sales person make one more sales call per week.

In the particular study that I read about, without going into all the particulars, one extra sales call per week, or essentially 45 sales calls per year, produced an incremental sales increase per representative of about $\$ 2.3 \mathrm{~m}$.

So, saving an hour or two a week has profound implications in terms of overall sales productivity and revenue for sales people. That would be really great to quantify in your user base.

IP: Yes. That's great.

MM: The next category of return is around data integrity and data quality. I'm sure that there's going to be some outsourcing service that comes in and just cleans up your contact prospect and customer database.

But I suppose the larger value in that would be the hours saved in terms of when a salesperson leaves. How much time it takes a new salesperson to come up to speed with respect to those existing accounts. By being able to archive the e-mail threads as well as the documents in particular accounts, that creates an audit trail of the conversation and would allow a sales person to come up to speed more quickly.

Again, the metric there I would think would be interesting to validate. It would be the amount of time it takes to come up to speed on a new account. That would be a certain number of account research hours, and the incremental sales being able to come up to speed more quickly on an account and/or the number of lost sales prevented because I was able to essentially recap the ongoing conversations.

The next thing that seems to me to be the ROI is around this whole notion of appointment synchronization. In and of itself, it's a kind of nonstarter. But the real value in that is the number of missed appointments per salesman per year avoided or precluded as a function of this tighter synchronization.

IP: That's a good point.

MM: Another aspect of a sales operation is really three things. One: overall pipeline in terms of how long it takes to move things through. So, pipeline acceleration. Being able to facilitate a buying process much more quickly as a function of speedier, tighter communication loops with buyers. That's definitely one ROI.

Another one is sales effectiveness. You can measure sales effectiveness in terms of the number of sales calls it takes to get a particular piece of business done. And then of course simply counting the revenue produced by that sales person - and revenue per sale divided by the number of sales calls would be another way.

Finally, the other part of a sales pipeline is the ability to accommodate new prospects into the sales pipeline. That's more the width of the pipeline. How many deals can a salesperson manage?

Typically, a salesperson really can't manage any more than 20 or 30 deals at any one given point in time because of the communication and interaction and collaboration it takes to keep those deals moving forward. So it seems to me that a more efficient and effective way of communicating and interacting with clients would essentially be to allow a salesman to manage $X$ number of more deals. It would be fascinating to understand how many more deals a salesperson could manage as a function of these automation tools. And as a function of being able to manage more deals, how much of 
that translates into incremental business as a function of greater efficiency and effectiveness.

Then finally, better forecasting as a function of having this deal oftentimes allows me to then understand where in the sales pipeline I can put scarce resources to further facilitate the buying process.

One of the most interesting things about the sales pipeline is that it divides into three sections. Prospecting, nurturing and closing. One of the interesting findings from the Miller and Heinman people of Strategic Selling indicates that most sales organizations spend too much time in the closing part of the buying process. That leads to wicked ups and downs in terms of revenues on a month-to-month basis.

It seems to me a better pipeline analysis and not just a pipeline analysis, but a better visibility into who's doing what with whom at what stage of the process.

Or supplementing that particular pipeline with individuals and/or external resources that can provide more prospecting capabilities and/or more sales-nurturing capabilities.

So it seems to me that those are the quantifications for the value proposition in a sales operation. I know I said a lot there, but do you have anything to add?

IP: You are absolutely right. Another aspect is that our SalesAlerts tool enables sales managers to stay tuned, and save a lot of their time they normally spent on phone calls and e-mails trying to find out what is going on with their sales reps' accounts, not to mention that receiving real-time updates, managers can react immediately, and never lose a single opportunity. MM: In terms of a sales operation, it seems to me that one of the things you begin to interact with starts with addressing basic productivity of knowledge workers in a sales role. What have you found in terms of systems or processes to increase the productivity of knowledge workers in sales roles?

IP: Well, there are fundamental values to making users more productive as individuals and in groups. And many modern technologies including all of those Web 2.0 products - are taking shots at that. There are, however, also psychological things. For example, resistance to change, and the search for personal value.

Whenever we evaluate a new technology, we ask ourselves how it's going to make our life easier and how it's going to make us more effective. If we look at it more closely, in order to make users want to use an application, we have to minimize the change in their work habits, and provide instant value that will make them like the new application.

The smallest change we can impose on users is no change at all. If we can give them a way to work with corporate applications while changing almost nothing about their work habits, I think that would solve the problem. Then we need to help them be more successful in a very immediate and very visible way.

For example, cut the routine work. Let them focus on sales. Not on double-entering data in all of those systems.

MM: Can you speak to the social dimension or the social life of sales information and sales support?

IP: Well, there are a few ways they can interact and share knowledge with each other. First, there is the CRM system. There is a knowledge repository object. They can store all their documents there. That's all the sales techniques and other useful documents. Once they put a new document there, an alert advises all our sales managers that a new document was added, and other users can immediately leverage that knowledge.

MM: As we move toward the conclusion of this interview, perhaps you can provide some forward-looking comments as far as how you see Sales Force automation evolving, and what sort of trends will lead to what kinds of breakthroughs.

IP: More and more business applications are built online. I think that the next big thing coming is virtual desktop. Still, it cannot substitute for the real desktop environment. People that usually travel a lot need to have an opportunity to work offline, when needed. Especially if they work in fields such as sales, perhaps.

Also, the move to web for big enterprises is not inexpensive. It takes at least several months. Migration costs for multiple systems at a large enterprise can run in the tens of millions of USD. So the next generation of composite applications and enterprise mashups for integration will be evolving.

They will provide capabilities to not only integrate applications at the enterprise 
level, but also between enterprises if need be. Or be on multiple devices - including mobile phones.

MM: Could you speak for a moment in terms of how you envision applications such as CRM working in with mobile devices?

IP: Many CRM vendors already now provide that functionality. For example, Salesforce.com has a mobile application. You can synchronize the data between your mobile phone and the CRM system.

Also, InvisibleCRM can be a bridge between mobile phones and the CRM system to sync common data types such as contacts and calendar. MM: Excellent.

I want to thank you very much for spending the time with us today, Inna.

IP: My pleasure. 